\title{
Structural, energetic and electronic properties of intercalated boron-nitride nanotubes
}

\author{
S RADA $^{1, *}$, M RADA $^{2}$ and E CULEA ${ }^{1}$ \\ ${ }^{1}$ Department of Physics \& Chemistry, Technical University of Cluj-Napoca, Cluj-Napoca 400 020, Romania \\ ${ }^{2}$ National Institute for R\&D of Isotopic and Molecular Technologies, Cluj-Napoca 400 293, Romania
}

MS received 8 November 2010; revised 28 March 2012

\begin{abstract}
The effects of chirality and the intercalation of transitional metal atoms inside single walled BN nanotubes on structural, energetic and electronic properties have been considered in this paper. The thermodynamic stability of BN nanotubes can be improved by the intercalation of cobalt or nickel. BN nanotubes can behave like an ideal non-interacting hosts for these one-dimensional chains of metal atoms. Their electronic properties are insignificantly modified.
\end{abstract}

Keywords. Intercalated boron-nitride nanotubes; quantum chemical calculations.

\section{Introduction}

According to theoretical studies, carbon nanotubes are predicted to be either metallic or semiconductor, depending on helicity and diameter of the tube (Hamada et al 1992). Furthermore, the curvatures and geometry of the tube causes important deviations in their electronic properties and anomalous magnetic properties (Lu 1995).

Nanotubes can be doped endohedrally by atoms or radicals to get some interesting properties. Rubio et al (1996), have studied the properties of chains of potassium and aluminum in carbon and boron-nitride nanotubes. The smaller bond length for linear chain of $\mathrm{K}$ and $\mathrm{Al}$ atoms, as compared to value for a bulk $b c c(\mathrm{~K})$ and a bulk $f c c(\mathrm{Al})$, has been understood in terms of a reduction of the coordination number from the bulk system to the linear chain (Zhao et al 2002).

However, we expected that BN tubes serve as noninteracting hosts for incorporation of other metal atoms since their bandgaps are large. Then, considering that insulators are much less polarizable than metals and semimetals, it is expected that other metal atoms are intercalated in the inner region of the $\mathrm{BN}$ nanotubes.

The aim of this study was to determinate the structural, energetic and electronic properties of the pure/intercalated boron-nitride nanotubes. In order to examine these properties, we explored theoretical models. The effects of chirality and transitional metal $(\mathrm{Fe}, \mathrm{Ni}, \mathrm{Co})$ intercalation, inside of the single walled BN nanotubes are investigated. The analysis of

\footnotetext{
*Author for correspondence (Simona.Rada@phys.utcluj.ro, radasimona@yahoo.com)
}

electronic properties has been carried out by density of state plots.

\section{Methodology}

In this paper, various intercalated BN nanotubes with onedimensional chains of metal atoms, have been submitted to molecular-mechanics (Allinger 1977) and semiempirical molecular orbital calculations (Stewart 1989) at PM3 levels (Dewar et al 1985) using the Spartan'02 software.

To obtain the densities of state (DOS) of these nanotubes, Hückel tight-binding calculations by BICON-CEDIT package (Brandle et al 1997) have been performed.

\section{Results and discussion}

We consider the following types of the BN nanotubes: $(10,0)$, $(5,5)$ and $(7,3) \mathrm{BN}$ intercalated with one-dimensional chains of four metal atoms (figure 1).

\subsection{Thermodynamic and kinetic stability}

Table 1 shows comparative variation enthalpies of formation (per atom) of the doped boron nitrogen tubes with different atoms. The tubes doped with cobalt and nickel behave like ideal non-interacting hosts stabilizing the BN nanotubes whereas the iron atoms lead to instability of the tubes. It is worth noting that according to our results for all the considered tubes, the intercalation one-dimensional chains of cobalt or nickel in armchair BN nanotubes are more energetically favourable. 

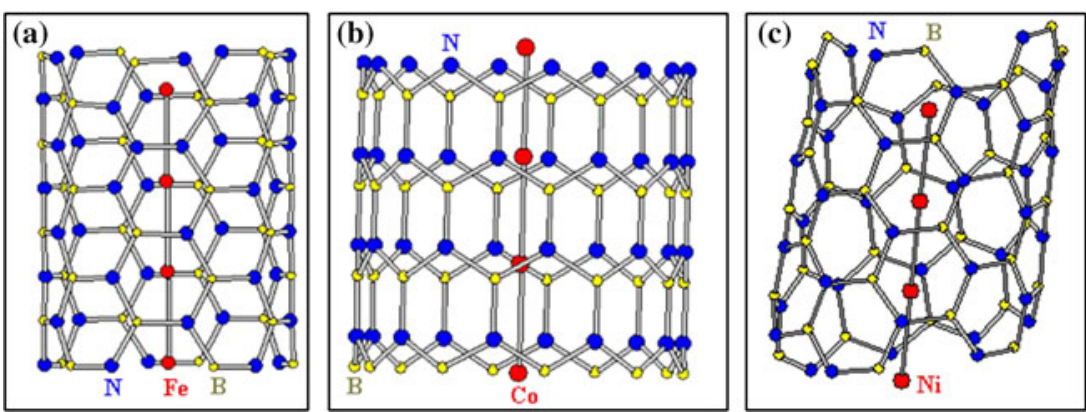

Figure 1. The structure intercalated boron-nitride nanotube with one-dimensional chains of metal atoms (Fe, Ni, Co): (a) armchair; (b) zigzag and (c) chiral tube.

Table 1. Calculations of the $\Delta \mathrm{H} /$ atom for intercalated $\mathrm{BN}$ nanotubes.

\begin{tabular}{lllll}
\hline & \multicolumn{4}{c}{$\Delta H(\mathrm{kcal} / \mathrm{mol}) /$ atom } \\
\cline { 2 - 5 } Tube geometry & Tube\&Co & Tube\&Ni & Tube & Tube\&Fe \\
\hline$(10,0)$ & -74.87 & -27.71 & $-15 \cdot 26$ & -13.04 \\
$(7,3)$ & -75.44 & -30.39 & -17.41 & -15.05 \\
$(5,5)$ & -79.7 & -32.09 & -22.05 & -17.89 \\
\hline
\end{tabular}

Table 2. Calculations of the $E_{\mathrm{HOMO}}-E_{\mathrm{LUMO}}$ for intercalated nanotubes.

\begin{tabular}{lcccc}
\hline & \multicolumn{4}{c}{$E_{\mathrm{HOMO}}-E_{\mathrm{LUMO}}(\mathrm{eV})$} \\
\cline { 2 - 5 } Tube geometry & Tube\&Fe & Tube\&Ni & Tube\&Co & Tube \\
\hline$(10,0)$ & 3.54 & 4.81 & 4.94 & $5 \cdot 57$ \\
$(7,3)$ & 2.05 & 3.96 & 3.61 & 4.28 \\
$(5,5)$ & 3.38 & 6.74 & 7.27 & 8.67 \\
\hline
\end{tabular}

The intercalated BN nanotubes attendance small values of their energy gaps $\left(E_{\mathrm{HOMO}}-E_{\mathrm{LUMO}}\right)$, (table 2$)$, so they are kinetically unstable relative to their pure analogues.

\subsection{Density of states}

One of the current research topics in this area is the influence of the different chiralities and a type of intercalated transition metal atom on electronic properties of the tubes. This includes the ability to use density of states. The results obtained for $(10,0) \mathrm{BN}$ nanotubes are revealed in figure 2 . From the DOS(E) plots, it is obvious that the lowest bands (valence band) are composed of $\mathrm{N}(2 s)$ derived states and the conduction bands corresponding to $\mathrm{N}(2 p)$ and $\mathrm{B}(2 s, 2 p)$ derived states, respectively.
The energy difference between the bottom of the conduction band and the top of the valence band in a semiconductor or an insulator is called the bandgap energy, $E_{\mathrm{g}}$. The bandwidth of the bandgap provides a distinction between a semiconductor and an insulator. When the bandgap is not very large, usually ranging from 0.5 to $3.0 \mathrm{eV}$, the material is a semiconductor, while an insulator has an $E_{\mathrm{g}}>$ $3 \mathrm{eV}$ (Greenham and Friend 1995; Dekker 1999; Rada and Silaghi-Dumitrescu 2006). So that energy gap is larger than $3 \mathrm{eV}$ and the $(10,0) \mathrm{BN}$ tube have insulator property. This result is in agreement with the ones predicted by Blasé et al (1999).

When chains of metal atoms are intercalated into the $(10,0) \mathrm{BN}$ nanotube, the electronic states in vicinity of the Fermi level can be significantly modified because of the new peaks associated with transitional metal atoms are found in conduction bands, figure $2(\mathrm{~b}-\mathrm{d})$. The valence bands are not affected. Instead of it, a stronger overlap in minimum conduction bands is predominantly composed of the orbital of $\mathrm{N}(2 p), \mathrm{B}(2 s, 2 p)$ and $\mathrm{Co}(3 d)$ or $\mathrm{Fe}(3 d)$. The density of states near the Fermi level consists of $\mathrm{Co}(3 d)$ states. This will play an important role in their magnetic properties (Hacohen et al 1998).

Intuitively, charge transfer from nanotube to metal can be understood by shift of Fermi level towards vacuum (Wilder et al 1998; Esfarjani et al 2003). Our calculations indicate that a weak charge transfer from the BN tube to metal is taking place because of the Fermi level shifts from $-13.37 \mathrm{eV}$ towards vacuum $(-13.21 \mathrm{eV}$ for tube\&Co). Then Fermi energy values confirm that $(10,0) \mathrm{BN}$ tube-Co interactions are stronger than the tube- $\mathrm{Fe}$ and the tube- $\mathrm{Ni}$, respectively. Our studies on electronic population analysis show that metal atoms act as acceptor electrons (negative charge on chains of metal atoms) and tube is donor of electrons subunit (positive charge), too.

On the basis of these studies it was concluded that metalmetal and metal-BN interactions are ionic and $\mathrm{Fe}, \mathrm{Ni}$ or Co act as electron acceptors. The BN nanotubes may be good hosts for the incorporation of these atoms because they behave like an ideal non-interacting hosts for the metal atoms inside and their electronic properties are insignificantly modified. 

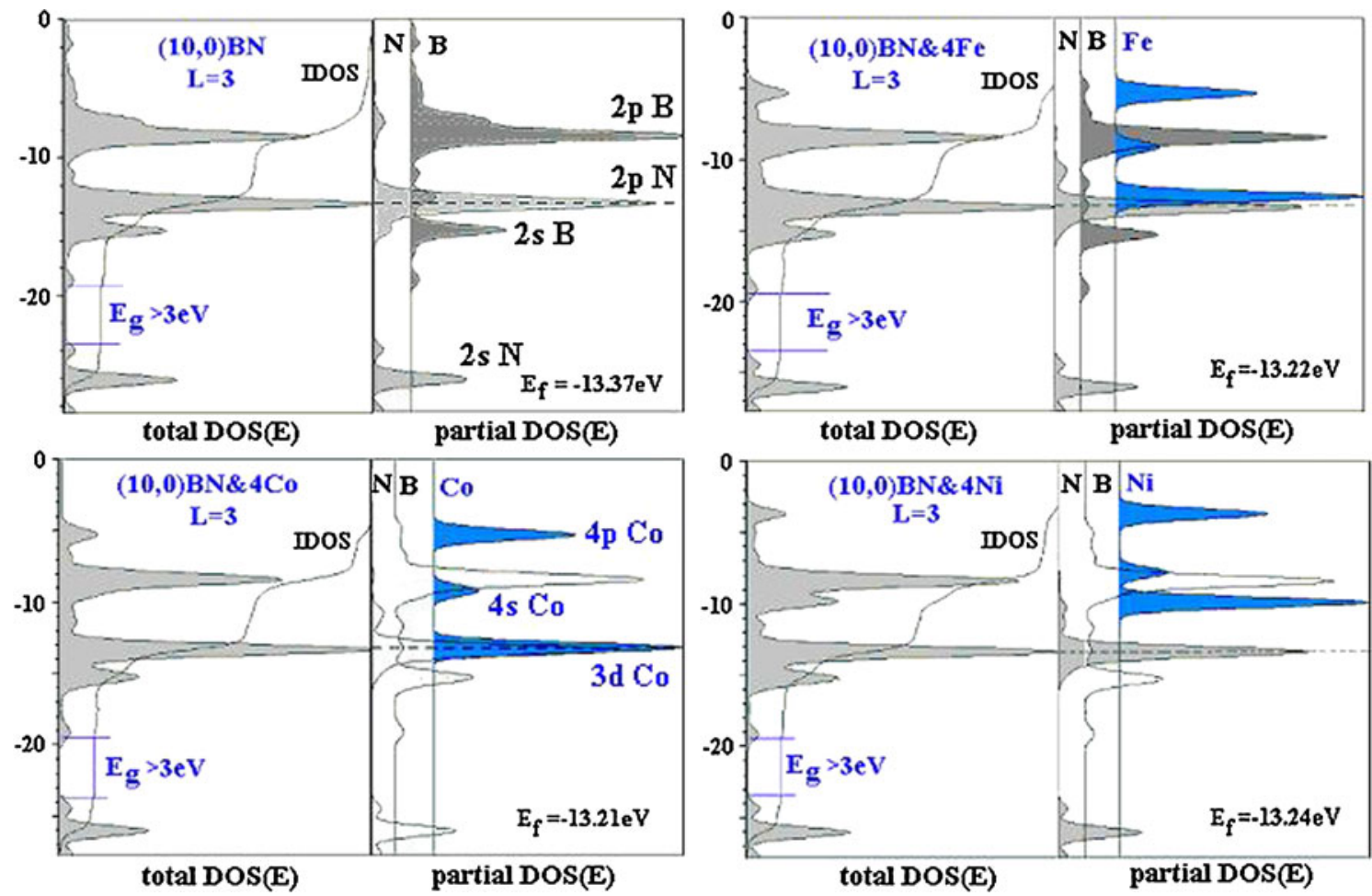

Figure 2. Total, partial DOS and IDOS diagrams for intercalated $(10,0) \mathrm{BN}$ tube.

\section{Conclusions}

According to our estimations, the intercalation onedimensional chains of cobalt or nickel in armchair BN nanotubes are more energetically favourable. Note also that the intercalated $\mathrm{BN}$ nanotubes behave like insulators independent on the chiralities of the tube and intercalated atom types.

\section{References}

Allinger N L 1977 J. Am. Chem. Soc. 998127

Blasé X, Charlier J C, De Vita A and Car R 1999 Appl. Phys. (Mater. Sci. Proc.) A68 293

Brandle M, Rytz R and Calzaferri G 1997 BICON-CEDIT manual, Bern

Dekker C 1999 Phys. Today 5222
Dewar M J S, Zoebisch E G, Healy E F and Stewart J J P 1985 J. Am. Chem. Soc. 1073902

Esfarjani K, Chen Z and Kawazoe Y 2003 Bull. Mater. Sci. 26105

Greenham N C and Friend R H 1995 Solid state physics, advances in research and application (eds) $\mathrm{H}$ Ehrenreich and $\mathrm{F}$ Spaepen, 491 (New York: Academic Press)

Hacohen Y R, Grunbaum E, Tenne R, Sloan J and Hutchison J L 1998 Nature 395336

Hamada N, Sawada S and Oshiyama A 1992 Phys. Rev. Lett. 68 1579

Lu J O 1995 Phys. Rev. Lett. 741123

Rada S and Silaghi-Dumitrescu I 2006 Revue. Roum. Chim. 51141

Rubio A, Miyamoto Y, Blasé X, Cohen M L and Louie S G 1996 Phys. Rev. 53B 4023

Spartan'02, Wavefunction Inc., Irvine, CA 2002

Stewart J J P 1989 QCPE Bulletin 980

Wilder J W G, Venema L C, Rinzler A G, Smalley R E and Dekker C 1998 Nature 3916662

Zhao J, Han J and Lu J P 2002 Phys. Rev. 65B 193401 